The Astrophysical JouRnal, 487:370-379, 1997 September 20

(C) 1997. The American Astronomical Society. All rights reserved. Printed in U.S.A.

\title{
HIGH-ENERGY RADIATION FROM RAPIDLY SPINNING PULSARS WITH THICK OUTER GAPS
}

\author{
L. Zhang AND K. S. ChENG \\ Department of Physics, University of Hong Kong, Pokfulam Road, Hong Kong; zhangl@hkuphy1.hku.hk, hrspksc@hkucc.hku.hk \\ Received 1996 December 20; accepted 1997 April 30
}

\begin{abstract}
We propose a self-consistent mechanism to estimate the size of the acceleration region in the outer magnetosphere of pulsars (outer gap) and calculate the high-energy radiation produced by the synchrocurvature mechanism from the outer gap. We find that a power-law energy distribution of the accelerated particles can be obtained if the outer gap is thick enough that $\boldsymbol{E} \cdot \hat{\boldsymbol{B}}$ inside the gap can be approximately proportional to $(\Omega r / c)^{1 / 2} B(r)$. We apply our model to explain X-rays and $\gamma$-rays from Geminga and PSR B1055-52, whose outer gaps may occupy $\sim 70 \%$ of the outer magnetosphere region. If the radius of curvature near the light cylinder of the medium outer gap is larger than the dipolar structure, then perhaps this model may also apply to PSR B1951+32, PSR B1706-44, and others.

Subject headings: MHD — pulsars: general — radiation mechanisms: nonthermal stars: magnetic fields
\end{abstract}

\section{INTRODUCTION}

Eight rapidly spinning pulsars with periods from 33 to $237 \mathrm{~ms}$ have been observed to emit high-energy $\gamma$-rays by EGRET (Nel et al. 1996); four of them are older than $10^{5} \mathrm{yr}$. Most of these $\gamma$-ray pulsars are also X-ray pulsars (Ögelman 1993; Becker \& Trümper 1993; Becker \& Trümper 1997). Theoretically, it is suggested that high-energy $\gamma$-rays are produced by the radiation of charged particles that are accelerated in the pulsar magnetosphere. There are two kinds of theoretical models: one is the polar gap model (e.g., Harding 1981), and another is the outer gap model (Cheng, Ho, \& Ruderman 1986a, 1986b, hereafter CHR I, CHR II; Ho 1989; Chiang \& Romani 1992; Chiang \& Romani 1994). Both models predict that electrons and positrons are accelerated in a charge depletion region called a gap by the electric field along the magnetic field lines and assume that charged particles lose their energies via curvature radiation in both polar and outer gaps.

According to the outer gap model, there are two types of pulsars: one is a Crab-type pulsar, and another is a Velatype pulsar. Although pair production and radiation mechanisms of these two types of pulsars are very different, it is assumed for both of them that primary radiation cannot be observed directly and that it acts only as an intermediate process to produce the observed radiation. In other words, the observed radiation is produced by the synchrotron radiation (Vela type) and synchrotron selfCompton mechanism (Crab type) of secondary $e^{ \pm}$pairs. This is true for the younger pulsars. However, Ruderman \& Cheng (1988) pointed out that as the period of the pulsar increases, the fractional volume of the outer gap will grow in order to maintain the required outer magnetosphere $e^{ \pm}$ production until finally all the available volume is used for the outer gap. This means that the size of the outer gap for older pulsars is larger. In this case, the approximation of the thin outer gap that implies a monoenergetic distribution of the primary $e^{ \pm}$pairs is no longer valid. Furthermore, Cheng \& Zhang (1996, hereafter Paper I) have pointed out that the radiation from the charged particles in the curved magnetic field should be described more accurately by the synchrocurvature mechanism, in which radiation is being emitted by charged particles moving in a spiral trajectory along a curved field line. It is noted that this mechanism differs from synchrotron and curvature mechanisms in general but reduces to either synchrotron radiation or curvature radiation in some parametric regions.

Therefore, older pulsars are expected to have thick outer gaps. Such a thick outer gap can be estimated by a selfsustained outer gap mechanism that will be described in $\S 2$ (see also Cheng 1994 and references therein). Charged particles moving in the curved field of the outer gap will be accelerated rapidly and reach a steady state within a very short period, and the energy distribution of the accelerated particles will not be monoenergetic because the steady state energies of the charged particles depend on the strength of the local electric field. In the thick outer gap, the local electric field could vary substantially.

This paper is organized as follows. In $\S 2$, we discuss why mature pulsars younger than $10^{6} \mathrm{yr}$ can still be $\gamma$-ray emitters. In $\S 3$, we describe a self-sustained outer gap mechanism for the mature pulsars, determine the size of the outer gap, and derive the energy distribution of the accelerated particles. In $\S 4$, we describe the hard X-ray spectrum emitted from the backflow of $e^{ \pm}$pairs. In $\S 5$, we present the primary radiation spectrum produced by synchrocurvature radiation of accelerated particles in the thick outer gap. In $\S 6$, we compare our model results with the observed spectra of $\gamma$-ray pulsars older than $10^{5} \mathrm{yr}$ whose outer gaps should occupy a large fraction of the outer magnetosphere. Finally, $\S 7$ we discuss whether this model can apply to young pulsars with a medium outer gap.

\section{WHY CAN MATURE PULSARS STILL EMIT $\gamma$-RAYS?}

A semianalytical outer gap model of rapidly spinning pulsars has been constructed by Cheng et al. (1986a, 1986b). In this model, it is assumed that large regions of the magnetospheric charge depletion (gaps) result from a global current flow pattern through the magnetosphere. In these gaps, there exist large electric fields along the magnetic field lines $(\boldsymbol{E} \cdot \hat{\boldsymbol{B}} \neq 0)$, which will accelerate the charged particles that are likely to be electrons/positrons to extreme relativistic energies. Sufficient $e^{ \pm}$pair production is sustained to short out $\boldsymbol{E} \cdot \hat{B}$, except in an almost slablike volume (the outer gap), and maintain the huge magnetospheric current 
flow through various mechanisms, including curvature radiation, inverse Compton scattering, and photon-photon pair production. According to CHR I and CHR II, the pair production and radiation mechanisms in the outer gap for the Crab pulsar and the Vela pulsar are very different, which are typical examples of Crab-type and Vela-type outer gaps, respectively. For Crab-type pulsars, primary photons are produced by curvature radiation and are converted into secondary $e^{ \pm}$pairs in collisions with X-rays, which are produced by the same secondary $e^{ \pm}$pairs via synchrotron radiation. These secondary pairs can prevent the further growth of the outer gap and produce low- to high-energy $\gamma$-rays through inverse Compton scattering with the same $\mathrm{X}$-rays. The observed $\mathrm{X}$-rays and $\gamma$-rays from Crab-type pulsars are a combination of secondary synchrotron radiation and inverse Compton scattering (CHR II; Ho 1989; Cheng \& Wei 1995). For Vela-type pulsars, the primary photons are produced by inverse Compton scattering of the primary $e^{ \pm}$pairs on IR photons and then produces secondary $e^{ \pm}$pairs in collisions with the same IR photons. The synchrotron radiation of these secondary $e^{ \pm}$ pairs produce crossed fan beams of secondary $\gamma$-rays and weaker ones of X-rays, which will be observed. The tertiary $e^{ \pm}$pairs with lower energies and a large flux are created in the region that is much farther from the outer gap by the collision of secondary $\gamma$-rays with secondary $\mathrm{X}$-rays and will fill much of the outer magnetosphere. The tertiary IR synchrotron radiation from the tertiary pairs causes the initial primary inverse Compton scattering in the gap and converts the scattered $\gamma$-rays and thus initiates the entire series of pair production processes. The observed $\gamma$-ray spectra exhibit a high-energy spectral break and a low-energy spectral break that are related to the maximum and the minimum energies of the secondary pairs. The former is one-half of the maximum energy of the primary photons, which is limited by radiation reaction of the primary electrons/positrons. The latter is explained in terms of the finite lifetime effect of the secondary $e^{ \pm}$pairs (CHR II), which gives the lower spectral break of $\sim 1 \mathrm{MeV}$. However, the observed spectral break for mature $\gamma$-ray pulsars seems to be $\sim 10-10^{2} \mathrm{MeV}$.

It should be pointed out that the outer gap is assumed to be a thin slab and the primary photons have monoenergetic distribution, which should be valid for young pulsars such as Crab-type pulsars, because the fractional volume of the outer magnetosphere occupied by the outer gap is only about 0.1. Ruderman \& Cheng (1988) estimated that the upper spectral break will eventually reach the lower spectral break when the star slows down sufficiently; then it would no longer be possible to maintain the bootstrapped production of $e^{ \pm}$pairs in a pulsar magnetosphere. From this argument, the self-sustained outer magnetosphere accelerator should be terminated when $P=P_{c}$, where $P$ is the period of the pulsar and $P_{c}$ is a critical period when the accelerator is terminated; $P_{c} \sim 0.13 \mathrm{~s}$ for Vela-type pulsars by assuming that all Vela-type pulsars have same inclination angle. This implies that the outer gap model in CHR I and CHR II is not valid for the pulsar with a period of greater than $0.13 \mathrm{~s}$. Furthermore, the size of the outer gap must increase as the pulsars evolve, which results in a question of validation of thin gap approximation for mature pulsars.

In order to explain $\gamma$-rays from mature pulsars such as Geminga in the outer gap model, Chen \& Ruderman (1993) reexamined the death line of the outer magnetospheric accelerator by taking into account the effect of inclination and concluded that pulsars with the same surface magnetic field but with a large inclination angle can survive as $\gamma$-ray pulsars longer than those with a smaller inclination angle. Based on this idea and the outer gap model, Cheng \& Ding (1994) investigated the evolution of the $\gamma$-ray spectra of Vela-type pulsars with different inclination angles and gave the period range of Vela-type pulsars. They showed that the $\gamma$-ray spectrum from the thin outer gap should be given by

$$
F_{\gamma}\left(E_{\gamma}\right) \propto \frac{1}{E_{\gamma}} \int_{\gamma_{\min }}^{\gamma_{\max }} d \gamma \gamma^{-2} \ln \left(\frac{\gamma_{\max }}{\gamma}\right) F(y),
$$

where $y=E_{\gamma} / \hbar \gamma^{2} \omega_{B}$ and $\gamma_{\max }$ and $\gamma_{\min }$ are the maximum and the minimum Lorentz factors of the secondary $e^{ \pm}$pairs, given by

$$
\begin{aligned}
\gamma_{\max } \sim \frac{m c^{2}}{E_{\mathrm{IR}}} & \sim m c^{2} \frac{\omega_{B}^{3}}{\hbar}\left(\frac{m c^{2} \Omega}{e^{2}}\right)^{-2}, \\
\gamma_{\min } & \sim \frac{\Omega m c^{3}}{e^{2} \omega_{B}^{2} \sin ^{2} \theta},
\end{aligned}
$$

where $E_{\mathrm{IR}}$ is a typical energy of IR photons, $\omega_{B}=(e B / m c)$, $B(r)=B_{s} R^{3} / r^{3}$ for a typical dipole field, $r$ is the typical distance to the outer gap, and $R$ is the stellar radius. The above spectrum can even fit mature pulsars, like Geminga, PSR B1055-52, and others. Although mature pulsars with larger inclination angles may still sustain the Vela-type outer gap mechanism and the model $\gamma$-ray spectrum may still fit the observed spectrum very well (Cheng \& Ding 1994), the required IR photon intensity seems unreasonably high. A more quantitative discussion will be given in $\S 6$. However, this does not mean that mature pulsars cannot have the self-consistent outer gap solution and are not efficient $\gamma$-ray emitters. In fact, Cheng (1994) proposed another self-sustained outer gap mechanism, in which low-energy $\mathrm{X}$-ray photons can replace the role of IR photons when IR photons are no longer available.

According to Cheng (1994), the energy of the primary $e^{ \pm}$ pairs will be limited by curvature radiation if the intensity of IR photons is not sufficiently high in the outer gap. After leaving the outer gap, half of the primary $e^{ \pm}$pairs will move toward the star and eventually strike the stellar surface. Although part of the energy of the primary particles will be lost on the way to the star via curvature radiation, about $10.6 P^{1 / 3}$ ergs per particle will still remain and eventually be deposited on the stellar surface. This energy will be emitted as X-rays from the stellar surface (such a process has been described by Halpern \& Ruderman 1993). Despite the very low X-ray photon density, about $10^{5}$ photons will be produced by every pair produced by X-rays and curvature photons in the gap. Such a huge multiplicity effect can produce sufficient $e^{ \pm}$pairs to sustain the gap as long as the center of mass energy of X-rays and curvature photons is greater than the threshold energy of $e^{ \pm}$pairs, which also determines the size of the outer gap. Any fluctuation of the gap size can be stabilized by the above condition. An increase of the gap size will increase the X-ray flux, and the overproduced pairs can reduce the gap size. Similarly, an decrease of the gap size will produce insufficient pairs and results in an increase of the gap size. Therefore, mature pulsars may still be efficient $\gamma$-ray emitters. 
Furthermore, Cheng (1994) estimated the size of the outer gap by comparing model results with the observed X-ray luminosities from the pulsars and showed that the size of the outer gap will be enlarged as the pulsar evolves; e.g., the fractional volume of outer magnetosphere occupied by the outer gap of Geminga is $\sim 0.76$. Obviously, this indicates that the approximation of the thin outer gap is no longer valid for mature pulsars. In other words, the energy distribution of the primary $e^{ \pm}$pairs is not monoenergetic. On the other hand, the $e^{ \pm}$pairs moving in the thick outer gap will be accelerated rapidly and reach a steady state in a very short time (e.g., Cheng, Zhang, \& Zhang 1996, hereafter Paper II), but the energy of the primary $e^{ \pm}$pairs will depend on the local electric field. The primary radiation produced by the primary $e^{ \pm}$pairs can no longer be described by a monoenergetic curvature spectrum because of the large region of the outer gap for the mature pulsars. Therefore, it is necessary to construct a thick outer gap model.

\section{THICK OUTER GAP MODEL}

Based on the self-sustained outer gap mechanism proposed by Cheng (1994), we will describe a thick outer gap model here; the schematic figure for the gap closure mechanism is shown in Figure 1. The amount of thermal X-rays in the polar gap is controlled by the return current produced by the primary $e^{ \pm}$pairs striking the stellar surface. Part of these thermal X-rays will emit toward the outer gap, whose size is controlled by pair production that results from the interaction of the thermal X-rays from the stellar surface with primary $\gamma$-rays from the outer gap. The detail structure of the thick outer gap is very complicated, but some simulations of plasma distribution about a rotating magnetized neutron star have been done (e.g., Krausse-Polstorff \& Michel 1985, who showed that $E_{\|} \sim E_{\perp}$ ). Therefore, we will give a simple estimation of the size of the outer gap. According to CHR I, about half of the primary $e^{ \pm}$pairs that are produced and separated by a local electric field in the outer gap will move toward the star and eventually strike the stellar surface. The return particle flux can be approximated

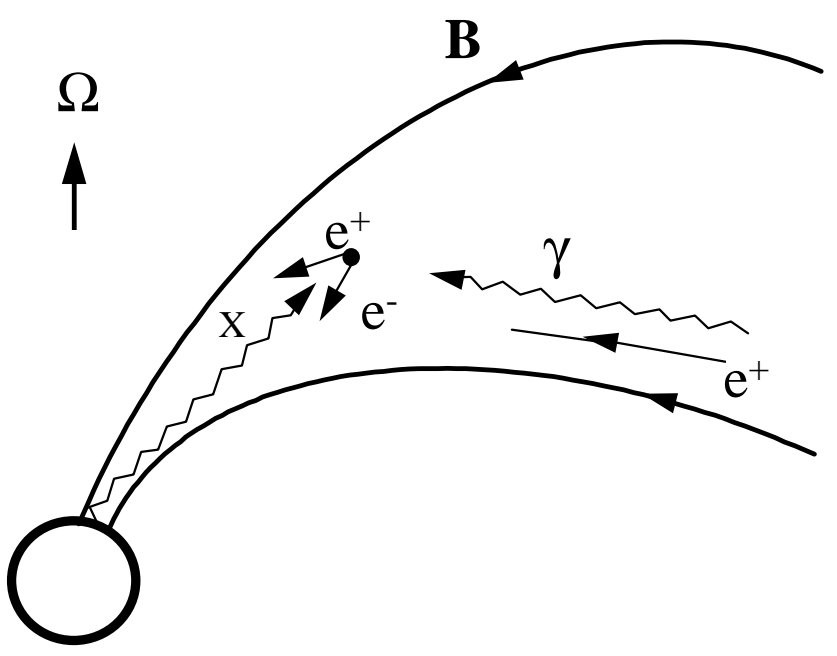

FIG. 1.-Gap closure mechanism. The return current $\left(e^{+}\right)$will heat the stellar surface and emit thermal X-rays. Part of these thermal X-rays will be emitted toward the outer gap. The size of the outer gap is controlled by $e^{ \pm}$pair production resulting from the interaction of thermal X-rays with primary $\gamma$-rays. by

$$
\dot{N}_{e}=\frac{1}{2} f \dot{N}_{\mathrm{GJ}},
$$

where $f$ is the fractional volume of the outer magnetosphere occupied by the outer gap and $\dot{N}_{\mathrm{GJ}}$ is the Goldreich-Julian particle flux (Goldreich \& Julian 1969), which is

$$
\dot{N}_{\mathrm{GJ}}=2 \pi r_{p}^{2} \frac{\Omega B(R)}{2 \pi c e} c \approx 2.7 \times 10^{30} B_{12} P^{-2} R_{6}^{3},
$$

where $r_{p}=R(\Omega R / c)^{1 / 2}$ is the radius of the polar cap, $P=2 \pi / \Omega$ is rotation period in seconds, $R_{6}$ is the radius of the star in units of $10^{6} \mathrm{~cm}$, and $B_{12}$ is the surface field in units of $10^{12} \mathrm{G}$. According to Halpern \& Ruderman (1993), each primary $e^{ \pm}$pair carries $10.6 P^{1 / 3}$ ergs onto the stellar surface. Therefore, the luminosity of X-rays from the stellar surface resulting from the bombardment of the return current is given by

$$
L_{X}=10.6 P^{1 / 3} \dot{N}_{e} \approx 1.4 \times 10^{31} f B_{12} P^{-5 / 3} \mathrm{ergs} \mathrm{s}^{-1} .
$$

There are two components of the X-rays: a hard component and a soft component. The hard component results from heating of the polar cap by $e^{ \pm}$pairs coming from the inner ends of the outer gap, which has a characteristic temperature of

$$
T_{h}=\left[\frac{10.6 p^{1 / 3} \dot{N}_{e}}{2 \pi f r_{p}^{2} \sigma}\right]^{1 / 4} \approx 3.8 \times 10^{6} P^{-1 / 6} B_{12}^{1 / 4} R_{6}^{3 / 4} \mathrm{~K},
$$

where $\sigma=5.67 \times 10^{-5} \mathrm{ergs} \mathrm{cm}^{-2} \mathrm{~s}^{-1} \mathrm{~K}^{-4}$ is Stefan's constant. The corresponding characteristic energy is

$$
E_{X}^{h} \approx 3 k T=1.2 \times 10^{3} P^{-1 / 6} B_{12}^{1 / 4} R_{6}^{3 / 4} \mathrm{eV} .
$$

On the other hand, if there is large density of $e^{ \pm}$pairs near the neutron star surface, then the $\mathrm{KeV} \mathrm{X}$-rays from a hot polar cap will be reflected back to the stellar surface because of the cyclotron resonance scattering and eventually reemit soft thermal X-rays with a characteristic temperature of

$$
T_{s}=\left[\frac{10.6 p^{1 / 3} \dot{N}_{e}}{4 \pi R^{2} \sigma}\right]^{1 / 4} \approx 0.1 f^{1 / 4} P^{-1 / 4} R_{6}^{1 / 4} T_{h} ;
$$

the corresponding energy is

$$
E_{X}^{s} \approx 0.1 f^{1 / 4} P^{-1 / 4} R_{6}^{1 / 4} E_{X}^{h} .
$$

CHR I gave a detailed description of electrodynamics, radiation mechanisms, and charge production processes in the outer gaps. On average, the electric field, $E_{\|}$, along the magnetic field in the large outer gap can be approximated by

$$
E_{\|} \approx \frac{B a^{2} \Omega}{c s} \approx f^{2} E_{\perp}\left(\frac{R_{L}}{s}\right),
$$

where $s$ is curvature radius of the magnetic field line, $R_{L}$ is the radius of the light cylinder, and $E_{\perp} \sim \Omega r B(r) / c$ is the electric field perpendicular to the magnetic field. Hence, the electric potential in the large outer gap can be written as

$$
V=f^{2} V_{\text {tot }},
$$

where $V_{\text {tot }}$ is total potential drop. In such a gap, the $e^{ \pm}$pairs will be accelerated to extreme relativistic energies, and these accelerated pairs will produce primary radiation. On the other hand, in order to sustain the large outer gap, a number of $e^{ \pm}$pairs is required. The condition of pair pro- 
duction is

$$
E_{X} E_{\gamma}\left(1-\cos \theta_{X \gamma}\right) \geq 2\left(m_{e} c^{2}\right)^{2},
$$

where $\theta_{X \gamma}$ is the angle between the directions of X-rays and $\gamma$-rays. From equation (13), the minimum energy of $\gamma$-rays can be determined by

$$
\begin{aligned}
& \left(E_{\gamma}\right)_{\min } \approx \frac{\left(m_{e} c^{2}\right)^{2}}{E_{X}} \\
& \quad \times \begin{cases}\sim 2 \times 10^{8} P^{1 / 6} B_{12}^{-1 / 4} R_{6}^{-3 / 4} \mathrm{eV} & \text { for hard X-rays }, \\
\sim 2 \times 10^{9} f^{-1 / 4} P^{5 / 12} B_{12}^{-1 / 4} R_{6}^{-1} \mathrm{eV} & \text { for soft X-rays } .\end{cases}
\end{aligned}
$$

Since the primary charged particles are moving in a spiral trajectory along the $B$ field and the $B$ field is curved, the primary radiation is better described by synchrocurvature radiation instead of by simple curvature radiation. The total power of the synchrocurvature radiation is given by (Cheng \& Zhang 1996)

$$
P_{\text {syn-cur }}(\alpha, \gamma)=-\frac{e^{2} \gamma^{4} c Q_{2}}{12 r_{c}}\left(1+\frac{7}{r_{c}^{2} Q_{2}^{2}}\right),
$$

where $\alpha$ and $\gamma$ are the pitch angle and Lorentz factor of the particle in the curved field, $c$ is light speed, $\omega_{B}=e B / \gamma m c$, $B(r)=B_{s} R^{3} / r^{3}$ for a typical dipole field, $r$ is the typical distance to the outer gap, $R$ is the stellar radius, $\Omega_{0}=c \cos$ $\alpha / s$, and

$$
\begin{aligned}
& r_{c}=c^{2} /\left[\left(r_{B}+s\right) \Omega_{0}^{2}+r_{B} \omega_{B}^{2}\right], \\
& Q_{2}^{2}=\frac{1}{r_{B}} \\
& \times\left(\frac{r_{B}^{2}+s r_{B}-3 s^{2}}{s^{3}} \cos ^{4} \alpha+\frac{3}{s} \cos ^{2} \alpha+\frac{1}{r_{B}} \sin ^{4} \alpha\right),
\end{aligned}
$$

$r_{B}=\frac{\gamma m c^{2} \sin \alpha}{e B}$.

The characteristic energy of the synchrocurvature photon is

$$
\begin{aligned}
E_{\gamma}= & \frac{3}{2} \hbar c \gamma^{3} \frac{1}{s} \\
& \times \sqrt{\left(\frac{r_{B}}{s}+1-3 \frac{s}{r_{B}}\right) \cos ^{4} \alpha+3 \frac{s}{r_{B}} \cos ^{2} \alpha+\frac{s^{2}}{r_{B}^{2}} \sin ^{4} \alpha .} .
\end{aligned}
$$

We have shown that charged particles moving in the curved electric and magnetic field lines are accelerated rapidly to extreme relativistic energies and the accelerated particles reach a steady state in a very short period (Paper II). In the steady state, the energy gain of the accelerated particles equals the radiation loss of the accelerated particle; i.e.,

$$
e E_{\|} c=P_{\text {syn-cur }} \text {. }
$$

We need to emphasize that the above equation holds for arbitrary $r$. Furthermore, the synchrocurvature radiation is a combination of synchrotron and curvature radiations and can be reduce to either synchrotron radiation or curvature radiation in some parametric regions (Paper I). The spectrum is dominated by curvature radiation in the highenergy range and by synchrotron radiation in the low-energy range (see Paper II). As an approximation, therefore, we can use curvature radiation instead of synchrocurvature radiation to estimate the Lorentz factor of the primary $e^{ \pm}$pair, which corresponds to the minimum energy of $\gamma$-rays that is required by pair production. For curvature radiation, the total radiation power is

$$
P_{\text {cur }}=-\frac{2}{3} \frac{e^{2}}{c^{3}}\left(\frac{c^{2}}{s}\right)^{2} \gamma^{4}
$$

with characteristic energy

$$
E_{\gamma}=\frac{3}{2} \hbar \gamma^{3}\left(\frac{c}{s}\right) .
$$

Therefore, from equations (14), (18), (19), and (20), the size of the outer gap limited by the hard $X$-rays can be given by

$$
f_{h} \approx 1.6 P^{23 / 18} B_{12}^{-2 / 3},
$$

where $s=r=R_{L}=c / \Omega$ and $R_{6}=1$ have been used. Furthermore, the size of the outer gap limited by the soft X-rays can be given by

$$
f_{s} \approx 5.5 P^{26 / 21} B_{12}^{-4 / 7} \text {. }
$$

Obviously, the presence of soft X-rays implies a larger size of the outer gap; if observed soft and hard X-rays have a thermal origin, then the size of the outer gap should be $f \sim f_{h}$. However, Halpern \& Ruderman (1993) pointed out that most of the hard X-rays will be scattered back to the stellar surface and reemitted as soft X-rays. In the rest of the paper, we shall assume $f=f_{s}$. The $\gamma$-ray luminosity is simply given by

$$
L_{\gamma} \approx 3.6 \times 10^{31} f^{3} P^{-4} B_{12}^{2} \operatorname{ergs~s}^{-1} .
$$

Using equations (6), (22), and (23), we can estimate the luminosities of the $\mathrm{X}$-rays and $\gamma$-rays. In Table 1 , the comparison of the observed and expected X-ray luminosities is given for $\gamma$-ray pulsars.

So far, we have considered self-sustaining gap-closing mechanisms in which the size of the gap is limited by the $e^{ \pm}$ pairs produced by collisions between high-energy photons from the gap and soft X-rays resulting from the surface heating by the backflowing primary $e^{ \pm}$pairs. However, there is another natural source of soft X-rays: production by a cooling mechanism. If soft X-rays are dominated by a standard cooling mechanism, following the arguments given above, we can derive the size of the outer gap as

$$
f_{c} \approx 4.5 P^{7 / 6} B_{12}^{-1 / 2} T_{c 6}^{-2 / 3},
$$

where $T_{c}$ is the surface temperature of the star determined by the standard cooling model. According to standard cooling models without rapid cooling mechanisms, e.g., kaon condensation or a direct Urca process (e.g., Tsuruta 1992), neutron stars with ages of $\sim 10^{4}$ yr should have a surface temperature of $\sim 10^{6} \mathrm{~K}$, which is comparable to that produced by polar cap heating of soft X-rays. Therefore, either $f_{s}$ or $f_{c}$ will give a similar gap size. On the other hand, when the star is older than $10^{5} \mathrm{yr}$, the stellar temperature will drop to $10^{5} \mathrm{~K}$, but the backflow of the primary $e^{ \pm}$pairs can still maintain a stellar temperature of $\sim 5 \times 10^{5} \mathrm{~K}$. Finally, before concluding which mechanisms should control the size of the outer gap, we would like to make three more remarks. (1) When internal heating mechanisms are likely to exist (e.g., Shibazaki \& Lamb 1989; Cheng et al. 1992), the cooling temperature of the star 
TABLE 1

\begin{tabular}{|c|c|c|c|c|c|c|}
\hline Pulsar & $\begin{array}{l}\text { Period } \\
\text { (s) }\end{array}$ & $B_{12}$ & $\begin{array}{c}\text { Age } \\
\left(10^{5} \mathrm{yr}\right)\end{array}$ & $f$ & $\log L_{X}^{\text {obs a }}$ & $\log L_{X}^{\text {th }}$ \\
\hline \multicolumn{7}{|l|}{ Thin gap (Crab type): ............ } \\
\hline Crab $\ldots \ldots \ldots \ldots \ldots \ldots$ & 0.033 & 3.8 & 0.013 & 0.04 & 36.2 & 32.8 \\
\hline PSR B1509-58 ............. & 0.15 & 15.5 & 0.016 & 0.11 & 34.3 & 32.8 \\
\hline \multicolumn{7}{|l|}{ Medium gap (Vela type): ......... } \\
\hline PSR B1951 + $32 \ldots \ldots \ldots \ldots \ldots$ & 0.04 & 0.49 & 1.1 & 0.15 & 33.4 & 32.3 \\
\hline PSR B1706-44 ............. & 0.102 & 3.1 & 0.17 & 0.17 & 33.1 & 32.5 \\
\hline PSR B0833-45 ............... & 0.089 & 3.4 & 0.11 & 0.14 & 32.7 & 32.6 \\
\hline \multicolumn{7}{|l|}{ Thick gap (post-Vela type):...... } \\
\hline PSR B0656+14 $\ldots \ldots \ldots \ldots \ldots$ & 0.385 & 4.7 & 1.1 & 0.70 & 32.5 & 32.4 \\
\hline Geminga..$\ldots \ldots \ldots \ldots \ldots \ldots \ldots$ & 0.237 & 1.6 & 3.4 & 0.71 & 31.7 & 32.2 \\
\hline PSR B1055-52 ............ & 0.197 & 1.0 & 5.3 & 0.74 & 32.5 & 32.2 \\
\hline
\end{tabular}

${ }^{\mathrm{a}}$ From Becker 1995.

can be maintained as

$T_{c}= \begin{cases}3 \times 10^{5} \beta_{42}^{1 / 4} \dot{\Omega}_{-12}^{1 / 4} \mathrm{~K} & \text { for superfluid frictional heating } \\ 2 \times 10^{5} \alpha_{42}^{1 / 4} \tau_{5}^{-1 / 4} \mathrm{~K} & \text { for crust cracking }\end{cases}$

where $\beta_{42} \equiv I_{p} \omega_{\mathrm{cr}} / 10^{42} \mathrm{~g} \mathrm{~cm}^{2} \mathrm{~s}^{-1}, I_{p}$ is the moment of inertia of the crust, $\omega_{\mathrm{cr}}$ is the critical angular velocity lag, $\alpha_{42} \equiv \mathscr{B} \theta_{\mathrm{cr}}^{2} / 10^{42}$ ergs, $\mathscr{B}$ is the constant that describes the strength of the crust (Baym \& Pines 1971), $\theta_{\text {cr }}$ is the critical shear angle, and $\tau_{5}$ is the age of the star in units of $10^{5} \mathrm{yr}$. (2) Even without the internal heating mechanisms, Page (1994) pointed out that because of the uncertainty of nuclear interaction at a high density, the protons and/or neutrons may not form a pairing state in the core of neutron stars. If this occurs, the observed soft X-ray emission from Geminga, PSR 0654 + 14, and PSR 1055-52 may be that of cooling X-rays without any internal heating mechanisms. (3) Despite the lack of concluding evidence for determining the origin of soft X-rays from older pulsars (e.g., Geminga), different models will predict a rather similar surface temperature, which only affects the size of the outer gap by a factor of $\left(T_{s} / T_{c}\right)^{2 / 3}$ and does not alter the qualitative picture of our model. For a self-consistent picture, we will assume that the size of the outer gap is determined by equation (22).

\section{NONTHERMAL HARD X-RAYS EMITTED FROM BACKFLOWING $e^{ \pm}$PAIRS FROM THE OUTER GAP}

In this section, we will estimate to what extent hard $\mathrm{X}$-rays will be reflected back to the surface by secondary $e^{ \pm}$ pairs and the radiation spectrum of the secondary pairs. According to Halpern \& Ruderman (1993), a heated polar cap is responsible for X-ray emission from Geminga. Although new X-ray data from Geminga indicate the presence of hard X-rays, in favor of nonthermal emission (Halpern \& Wang 1997), it is generally believed that soft $\mathrm{X}$-rays have a thermal origin. Three possible origins of soft thermal X-rays exist: (1) Standard cooling of neutron stars with a standard neutron star structure (e.g., Sauls 1989; Wambach, Ainsworth, \& Pines 1991; Pines \& Alpar 1992), which requires heating mechanisms (Shibazaki \& Lamb 1989; Chong \& Cheng 1993; Cheng \& Wei 1995). (2) Standard cooling of neutron stars with a nonstandard neutron star structure in which neutron and proton pairing states do not exist or only one of them exists (e.g., Page 1994, 1995). (3) The process proposed by Halpern \& Ruderman (1993), as discussed in a previous section. Here we will focus on the third case.

The relativistic primary electrons/positrons in the outer gap have a Lorentz factor of

$$
\gamma_{e} \approx 3 \times 10^{7} f^{1 / 2} B_{12}^{1 / 4} P^{-1 / 4} R_{6}^{3 / 4} .
$$

These primary charged particles will radiate photons with typical energy (eq. [20]) given by

$$
E_{s c}=(3 / 2) \hbar(c / s) \gamma^{3} \approx 2 \times 10^{8} f^{3 / 2} B_{12}^{3 / 4} P^{-7 / 4} R_{6}^{9 / 4} \mathrm{eV} .
$$

Part of these photons, which will be estimated later, will be converted into secondary $e^{ \pm}$pairs in both open and closed magnetic field line regions near the neutron star because of magnetic pair creation (Halpern \& Ruderman 1993). The condition of these photons to make pairs is given by (Sturrock 1971; Ruderman \& Sutherland 1975)

$$
\frac{E_{\gamma}}{2 m_{e} c^{2}} \frac{B\left(r_{s}\right)}{B_{g}} \approx \frac{1}{15}
$$

where $B\left(r_{s}\right)$ is local magnetic field at position of $r_{s}$ and $B_{g}=$ $m_{e}^{2} c^{3} / e \hbar=4.4 \times 10^{13}$ G. From this condition and assuming that the local field is a dipole field, then the distance to the star can be given by

$$
\frac{r_{s}}{R} \approx 4.3 f^{1 / 2} P^{-7 / 12} B_{12}^{7 / 12} R_{6}^{3 / 4}
$$

or

$$
\frac{r_{s}}{R_{L}} \approx 10^{-3} f^{1 / 2} P^{-19 / 12} B_{12}^{7 / 12} R_{6}^{7 / 4}
$$

where $B_{12}=B_{s} / 10^{12}$ and $R_{6}=R / 10^{6}$. On the other hand, the power provided by the outer gap is given by equation (23). Therefore, the flux of $e^{ \pm}$produced at the distance $r_{s}$ can be expressed as

$$
\dot{N}_{e^{ \pm}}^{\prime}=\frac{P_{\text {gap }}}{E_{\text {cur }}}\left(\frac{r_{s}}{R_{L} / 2}\right)^{2} \frac{\pi}{\Delta \Omega},
$$

where $\Delta \Omega$ is the solid angle of the primary $\gamma$-ray beam. These relativistic $e^{ \pm}$pairs will emit high-energy photons toward the star where the magnetic field is increasing rapidly, and hence their energies are sufficiently high to make a higher generation of $e^{ \pm}$pairs and then form a cascade until its Lorentz factor approaches unity. The 
resulting flux of the pairs can be written as

$$
\dot{N}_{e^{ \pm}}^{(1)} \approx \frac{2 E_{s c}}{1 \mathrm{MeV}} \dot{N}_{e^{ \pm}}^{\prime} \approx 1.8 \times 10^{33} f^{4} P^{-19 / 13} B_{12}^{19 / 6} R_{6}^{19 / 2} \mathrm{~s}^{-1}
$$

Here, we have chosen $\Delta \Omega \sim 1$ sr. Furthermore, Halpern \& Ruderman (1993) argued that the primary electrons/ positrons will radiate most of their energies $\gamma_{e} m c^{2}$ (eq. [26]) during the motion from the inner boundary of the outer gap $\left(r_{i}\right)$ toward the neutron star surface (note that they still carry $10.6 P^{1 / 3}$ ergs when they strike the star and heat up the polar cap to emit thermal hard X-rays). Since most of these highenergy photons are emitted toward the star, they will be converted into $e^{ \pm}$pairs by the stellar magnetic field. Therefore, the number of secondary pairs created in this way is given by

$$
\begin{aligned}
\dot{N}^{(2)} \sim & \frac{\dot{N}_{e^{ \pm}} \gamma_{e} m c^{2}}{1 \mathrm{MeV}}\left(\frac{r_{s}}{f r_{i}}\right)^{2} \approx 3.8 \times 10^{37}\left(\frac{r_{s}}{f r_{i}}\right)^{2} \\
& \times f^{-3 / 2} B_{12}^{5 / 4} P^{-9 / 4} \mathrm{~s}^{-1},
\end{aligned}
$$

where $\dot{N}_{e^{ \pm}}$is given in equation (4) and the position of the inner boundary $\left(r_{i}\right)$ sensitively depends on the inclination angle $\chi$; e.g., $r_{i} \sim 4 R_{L} / 9 \tan ^{2} \chi$ (for $\tan \chi \gg 1$ ) in a dipolar field approximation. Here, we arbitrarily choose $r_{i} / R_{L} \sim$ 0.3 , which corresponds to an inclination angle of $\sim 50^{\circ}$. The number density of these pairs at the distance $r$ to the star can be estimated as $n_{e^{ \pm}}=\left(\dot{N}_{e^{ \pm}}^{(1)}+\dot{N}_{e^{ \pm}}^{(2)}\right) / 4 \pi r^{2} c$. For Geminga, $P=0.237 \mathrm{~s}$ and $B_{12}=1.6$, so we have $\dot{N}_{e \pm}^{(1)} \sim$ $10^{37} \mathrm{~s}^{-1}, \dot{N}_{e^{ \pm}}^{(2)} \sim 10^{38} \mathrm{~s}^{-1}$, and $n_{e^{ \pm}} \sim 4 \times 10^{14} \mathrm{~cm}^{-3}$ at the stellar surface, which is consistent with the estimation given by Halpern \& Ruderman (1993). The details of how these pairs reflect the polar cap X-rays follow.

The distribution of the hard thermal X-ray photons is assumed to be blackbody distribution, so the differential number density is given by

$$
n(\omega) d \omega=\frac{\omega^{2} d \omega}{\pi^{2} c^{3}\left(e^{\hbar \omega / k T_{h}}-1\right)},
$$

where $\omega$ is the angular frequency of the X-ray photons, $T_{h}$ is given by equation (7), and $T_{h} \approx 5.4 \times 10^{6} \mathrm{~K}$ for Geminga. These X-ray photons will be effectively scattered because of resonant cyclotron scattering by an $e^{ \pm}$plasma screen in strong magnetic field lines. The cross section is given by (Halpern \& Ruderman 1993)

$$
\sigma=\sigma_{\mathrm{T}}(\hat{\mathscr{E}} \cdot \hat{B})^{2}+\frac{2 \pi^{2} e^{2}}{m c}|\hat{\mathscr{E}} \times \hat{B}|^{2} \delta\left(\omega_{B}-\omega\right),
$$

where $\hat{\mathscr{E}}$ is the electric field polarization of the X-rays, $\sigma_{\mathrm{T}}$ is the Thomson cross section, and $\omega_{B}=e B(r) / m c$. Therefore, the absorption coefficient $\alpha(r)$ can be expressed as

$$
\alpha(r)=\int n_{e^{ \pm}} \sigma\left[\frac{n(\omega) d \omega}{\int_{0}^{\infty} n(\omega) d \omega}\right] .
$$

From equation (35), the cross section depends on the angle between the electric field polarization and the magnetic field polarization. Two extreme cases are (i) $\mathscr{E} \cdot \hat{B}=0$ and (ii) $\hat{\mathscr{E}} \times \hat{B}=0$. For the first case, the hard X-rays can escape from the polar cap because of a negligible optical depth (Halpern \& Ruderman 1993), but the hard X-rays will be effectively scattered for the second case. If the polar cap hard X-rays have a thermal origin, then the second case should be the dominant one; i.e., the hard X-rays are effectively scattered. In fact, new X-ray data from Geminga indicate that the hard X-rays may be nonthermal. In this case, expression (36) can be approximated by

$$
\alpha(r)=0.42 n_{e \pm}\left(\frac{2 \pi^{2} e^{2}}{m c \omega_{0}}\right)\left(\frac{\hbar \omega_{0}}{k T_{h}}\right)^{3} \frac{(r / R)^{-8}}{e^{\hbar \omega_{0} / k T_{h}(r / R)^{-3}}-1},
$$

where $\omega_{0}=e B_{\mathrm{s}} / m c \approx 1.8 \times 10^{19} B_{12} \mathrm{~s}^{-1}$. Hence, the optical depth of X-ray photons is given by

$$
\tau=\int_{R}^{r_{s}} \alpha(r) d r
$$

For Geminga, the absorption coefficient versus distance $r$ for different temperatures is shown in Figure 2. It can be seen that an optically thick cyclotron resonant backscattering layer is located at the position of $r \sim 2.6 R$ for $T=5 \times 10^{6} \mathrm{~K}$ and $\sim 5.2 R$ for $T=6 \times 10^{5} \mathrm{~K}$. Integrating equation (38) for Geminga's parameters, we have $\tau \sim 33 \gg 1$. Therefore, most of thermal X-rays with characteristic temperature $T_{h}$ would not escape and will be reflected back to the stellar surface. As mentioned by Halpern \& Ruderman (1993), although some of the surface radiation with lower temperature will be reflected at some distance (say, $\sim 6 R$ ), there is no significant further reduction in emitted soft X-ray energy because of thermal equilibrium. Finally, soft X-rays will escape.

Now we consider the radiation spectrum of X-rays. We believe that the synchrotron radiation of the secondary pairs will be responsible for the hard X-rays and the polar cap hard X-rays will be reflected back to the stellar surface to produce soft X-rays; i.e., soft X-rays have a thermal distribution and hard X-rays have a nonthermal one. For the soft $\mathrm{X}$-rays, the spectrum is

$$
N_{s X}=A_{s} \frac{\left(E_{\gamma} / m_{e} c^{2}\right)}{e^{E_{\gamma} / k T}-1},
$$

where $A_{s} \approx 10^{45} f^{4} P^{-19 / 13} B_{12}^{19 / 16} R_{6}^{19 / 2} T_{6}^{-4} \mathrm{KeV}^{-1} \mathrm{~s}^{-1}$ and $T_{6}$ is in units of $10^{6} \mathrm{~K}$. For the hard X-rays, assuming the distribution of the secondary pairs can be approximated by the $\delta$ function, then the steady state spectrum is given by

$$
\frac{d N_{e}}{d E_{e}} \approx \frac{\dot{N}_{e^{ \pm}}}{\dot{E}_{e}} \int \delta\left(E_{e}-\frac{E_{s c}}{2}\right) d E_{e} \propto\left(\frac{E_{e}}{m_{e} c^{2}}\right)^{-2},
$$

where $\dot{E}_{e}=9.8 \times 10^{-7} B^{2}\left(E_{e} / m_{e} c^{2}\right) \mathrm{KeV} \mathrm{s}^{-1}$. These secondary pairs will immediately lose their energies via synchro-

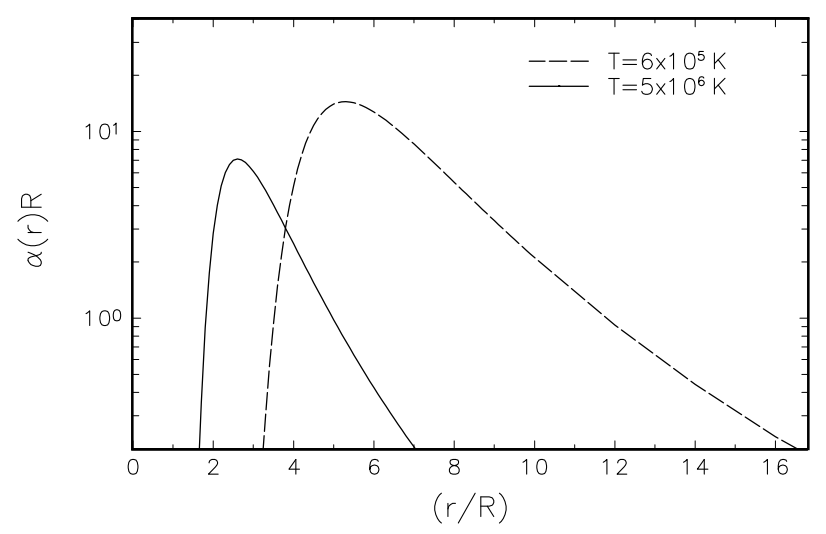

FIG. 2.-Absorption coefficient vs. $r$ for temperatures of $T=6 \times 10^{5}$ and $T=5 \times 10^{6} \mathrm{~K}$. 


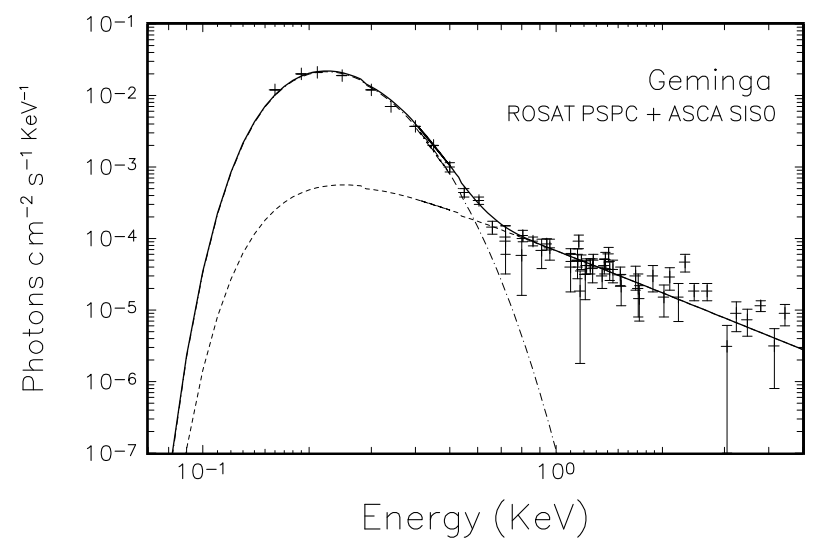

FIG. 3.-Comparison between the observed data and our model spectra for Geminga. The data are taken from Halpern \& Wang (1997).

tron radiation. Since these high-energy synchrotron photons are emitted toward the star where the magnetic field is increasing rapidly, they will be converted into $e^{ \pm}$ pairs, which will again radiate synchrotron photons. These electromagnetic processes will go on until the energy of the synchrotron photon is below $1 \mathrm{MeV}$. The energy spectrum of these $e^{ \pm}$pairs should be

$$
\frac{d N_{e}}{d E_{e}} \propto\left(\frac{E_{e}}{1 \mathrm{MeV}}\right)^{-3},
$$

and the synchrotron spectrum is given by

$$
N_{h X}=A_{h}\left(\frac{E_{\gamma}}{1 \mathrm{MeV}}\right)^{-2} \hbar \frac{e B\left(r_{s}\right)}{m c} \leq E_{\gamma} \leq 1 \mathrm{MeV}
$$

where

$$
A_{h} \approx \dot{N}_{e^{ \pm}}^{(2)} / \ln \left[1 \mathrm{MeV} / \hbar \frac{e B\left(r_{s}\right)}{m c}\right] \mathrm{KeV} .
$$

Figure 3 shows the comparison between the observed data and our model spectra for Geminga, where interstellar absorption has been taken into account (the interstellar medium photoelectric cross section is taken from Morrison \& McCammon 1983, and an interstellar column density of $N_{\mathrm{H}}=1.5 \times 10^{20} \mathrm{~cm}^{2}$ is used) and the distance to Geminga is taken from Caraveo et al. (1996). The dot-dashed line is the thermal spectrum with temperature $T_{s}$ given in equation (9), the dashed line is the power-law spectrum (eq. [42]), and the solid line is the sum of them. It seems that the observed data and model spectra are quite consistent.

\section{RADIATION FROM THE THICK OUTER GAP}

We have considered the motion of a relativistically charged particle in curved electric and magnetic field lines in detail, where three mechanisms of synchrotron, curvature, and synchrocurvature radiation have been taken into account (see Paper II). We have shown that a relativistically charged particle with an arbitrary pitch angle moving in the accelerator of outer magnetosphere will be accelerated rapidly to extreme relativistic energy and reach a steady state in a very short period (Paper II). As pointed out by Cheng \& Zhang (1996), a synchrocurvature mechanism can realistically describe the radiation from the relativistically charged particles in the pulsar magnetosphere. In fact, for given values of $B, E$, and $s$, the pitch angle in the steady state has a finite value for a given radiation mechanism. For example, for the typical parameters of the outer gap, $s=10^{8} \mathrm{~cm}, B=10^{6} \mathrm{G}$, and $E=10^{5} \mathrm{cgs}$, the Lorentz factors and pitch angles in the steady state are $\sim 3 \times 10^{7}$ and $\sim 10^{-3}$ for synchrocurvature radiation (Paper II). This means that the spectra of both curvature radiation and synchrotron radiation have important roles in the overall radiation spectrum from the primary $e^{ \pm}$pairs, where low-energy radiation is dominated by the synchrotron radiation and high-energy radiation is dominated by curvature radiation if the primary $e^{ \pm}$pairs have a power-law distribution.

Now we turn to the steady state energy distribution of the accelerated particle, which relates to the geometry of the outer gap in the magnetosphere of the pulsar. The curvature radius around the first open field line can be expressed approximately by $s \approx\left(r R_{L}\right)^{1 / 2}$, where $r$ is the distance along the field line to the star. For other open magnetic field lines, the curvature radius can be expressed approximately by (Arons \& Scharlemann 1979)

$$
s_{i} \sim \frac{\theta_{p}}{\theta_{i}} \sqrt{r R_{L}},
$$

where $\theta_{p}$ is the angular width of the polar cap and $\theta_{i}$ is the polar angle of the $i$ th field line. Therefore, the local electric field along the magnetic field lines can be expressed by equation (11) and rewritten as

$$
E_{\|}(r) \approx f^{2}\left(\frac{r}{R_{L}}\right)^{1 / 2} B(r)
$$

where $B(r)$ is the local magnetic field. In the outer gap, the local number density of the accelerated particles is given by

$$
n(r)=\frac{\overrightarrow{\boldsymbol{\Omega}} \cdot \overrightarrow{\boldsymbol{B}}}{2 \pi c e} \sim \frac{\Omega B_{s} R^{3}}{2 \pi c e} r^{-3}
$$

where $B_{s}$ and $R$ are the surface magnetic field and radius of the pulsar and $r$ is the distance to the pulsar. Hence, the particle numbers in the volume element of $\Delta V=2 s \alpha L(r) \Delta s$ can be expressed as

$$
\Delta N \approx n(r) \Delta V=2 s \alpha L(r) \Delta s n(r),
$$

where $2 s \alpha$ is a length unit along the magnetic field within which the relativistic particles with a pitch angle $\alpha$ can radiate in the same direction (cf. Fig. 4 ) and $L(r)$ is the width of the outer gap perpendicular to the plane of $(s, B)$, which is the function of the distance to the pulsar. On the other

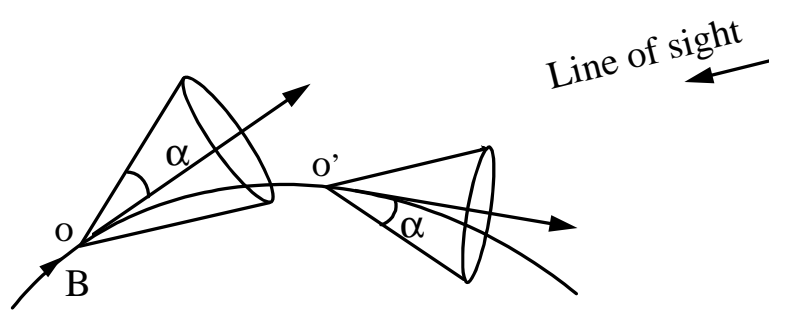

FIG. 4.-Radiation from relativistic particles with pitch angle $\alpha$ between $\mathrm{O}$ and $\mathrm{O}^{\prime}$ will be emitted into the same direction parallel to the line of sight. 
hand, the local energy of the accelerated particle and pitch angle in the steady state can be determined by solving the equation of motion of the charged particles in the specific position of the outer gap. The following approximated equilibrium solutions are obtained (Paper II):

$$
\begin{gathered}
\sin \alpha \approx \alpha \approx \eta \frac{\gamma m c^{2}}{e B(r) s}, \\
P_{\text {syn-cur }}(\alpha, \gamma)=e f^{2} c\left(\frac{r}{R_{L}}\right)^{1 / 2} B(r),
\end{gathered}
$$

where $\eta$ is of order 1 . The physical meaning of equations (47) and (48) corresponds to $\sin \alpha \sim$ (Larmor radius)/(local radius of curvature) and (energy gain) $=$ (energy loss), respectively. In order to simplify our calculation, we use a power law to fit the energy of primary particle (see Fig. 5) and find

$$
\gamma(x) \approx \gamma_{0} x^{-3 / 4}
$$

with

$$
\gamma_{0} \approx 2.0 \times 10^{7} f^{1 / 2} B_{12}^{1 / 4} P^{-1 / 4},
$$

where $x \equiv s / R_{L}$ and $s^{2} \approx r R_{L}$ have been used to derive the above expression. From equation (49), we have $d \gamma /$ $d x \propto \gamma^{7 / 3}$. Furthermore, the pitch angle can be approximated by

$$
\alpha \approx \frac{E}{e B S} \approx 0.79 f^{1 / 2} B_{12}^{-3 / 4} P^{7 / 4} x^{17 / 4},
$$

so equation (46) can be written by

$$
\frac{d N}{d x} \approx N_{0} g(x) x^{5 / 4}
$$

with

$$
g(x) \equiv \frac{L(r)}{r} \propto x
$$

it should be noted that $g(x) \propto x$ is strictly true only for an aligned rotator. Although the geometry of the outer gap for nonaligned rotation is more complicated, we shall assume that this relation still holds. As an approximation, we use

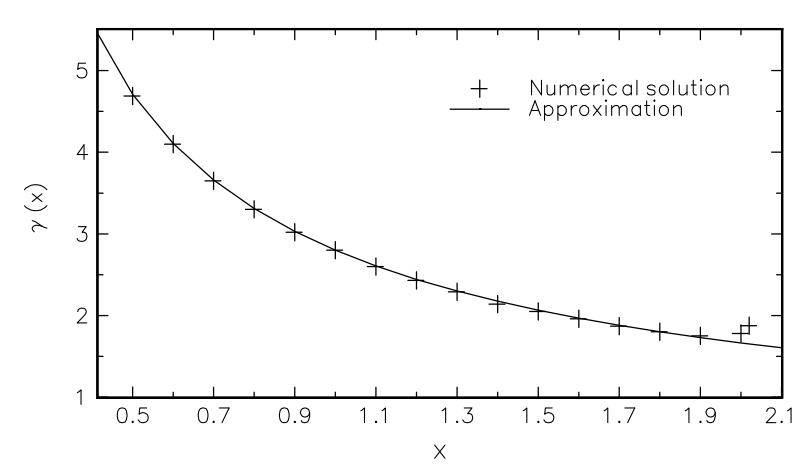

FIG. 5.-Lorentz factor of primary particles vs. $x$ for Geminga. A solid line represents the fitting result, and a plus sign represents the numerical results by solving eqs. (22) and (48), where $\gamma(x)$ is in units of $10^{7}$. $g(x)=x . N_{0}$ is a constant determined by

$$
\int_{x_{\min }}^{x_{\max }} N_{0} x^{9 / 4} d x \leq f \dot{N}_{\mathrm{GJ}} \frac{x_{\max }}{c} R_{L},
$$

where $x_{\max }$ is the maximum value of $x$ in the outer gap. Therefore, equation (52) can be expressed as

$$
\frac{d N}{d x} \approx 1.4 \times 10^{30} f B_{12} P^{-1}\left(\frac{x}{x_{\max }}\right)^{9 / 4} ;
$$

the corresponding energy distribution of the accelerated particles in a steady state is

$$
\frac{d N}{d E_{e}} \propto\left(\frac{\gamma}{\gamma_{0}}\right)^{-16 / 3},
$$

where $E_{e}$ is the energy of the accelerated particle. It should be noted that the Lorentz factor in equation (56) is the function of $x$ given by equation (49). For such a steady state energy spectrum of the accelerated $e^{ \pm}$pairs, using the general formula of radiation power spectrum given by Cheng \& Zhang (1996) and $\left(d N / d E_{e}\right) d E_{e}=(d N / d x) d x$, we can express the radiation spectrum from the thick outer gap as

$$
\begin{aligned}
\frac{d^{2} N_{\gamma}}{d E_{\gamma} d t} & =\frac{\sqrt{3} e^{2}}{2 h} \frac{1}{E_{\gamma}} \int_{x_{\min }}^{x_{\max }} \frac{d N(x)}{d x} \frac{\gamma}{r_{c}} \\
& \times\left[\left(1+\frac{1}{r_{c} Q_{2}^{2}}\right) F(y)-\left(1-\frac{1}{r_{c} Q_{2}^{2}}\right) y K_{2 / 3}(y)\right] d x,
\end{aligned}
$$

where $x_{\max }$ and $x_{\min }$ are maximum and minimum curvature radii of the magnetic field lines in units of radius of light cylinder, $y=E_{\gamma} / E_{c}^{\text {syn-cur }}, E_{c}^{\text {syn-cur }}$ is the characteristic energy of synchrocurvature radiation given by equation (17), and $F(y)=\int_{y}^{\infty} K_{5 / 3}(z) d z$, where $K_{5 / 3}$ and $K_{2 / 3}$ are the modified Bessel functions of orders $5 / 3$ and $2 / 3$.

Strictly speaking, the integration over $x$ in equation (57) is from the bottom of the gap to the top of the gap along a line whose tangents at any intersection point with magnetic field lines are parallel to the line of sight. For simplicity, we will assume that it is a straight line bound by the inner and outer boundaries of the outer gap; then $x_{\min }$ and $x_{\max }$ are determined by the first and the last open lines in the outer gap, respectively, which also relate to the inclination angle of the pulsar. For the pulsars with inclination angles of $\sim 50^{\circ}, x_{\min } \sim 0.3$. On the other hand, the value of $x_{\max }$ should be greater than 1 because $\theta_{p} / \theta_{f}>1$, where $\theta_{f}$ is the magnetic colatitude of the last open field line of the outer gap. In principle, $\theta_{f}$ decreases as the size of the outer gap increases; however, the details of the geometry of the outer gap are not clear, and here we use $x_{\max }$ as a parameter.

\section{APPLICATIONS}

In this section, we will compare our model results with the observed spectra of $\gamma$-ray pulsars. The expecting spectrum at the Earth is given by

$$
F\left(E_{\gamma}\right)=\frac{1}{\Delta \Omega d^{2}} \frac{d^{2} N_{\gamma}}{d E_{\gamma} d t}
$$

where $\Delta \Omega$ is the solid angle of $\gamma$-ray beaming and $d$ is the distance to the pulsar.

Therefore, there are three parameters in this model, namely, $\Delta \Omega, x_{\min }$, and $x_{\max }$, which depend on the geometry 


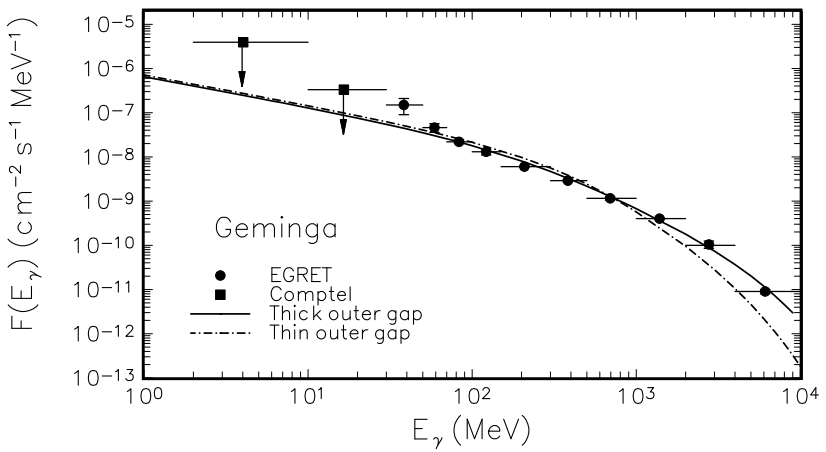

Fig. 6.-Comparison of model results of Geminga with observed data by COMPTEL (squares) (Bennett et al. 1993) and EGRET (circles) (MayerHasselwander et al. 1994; Fierro 1995). The solid curve represents the model result of the thick outer gap, and the dash-dotted curve represents the model result of the thin outer gap, which is taken from Cheng \& Ding (1994).

of the outer gap. From Table 1, the sizes of the outer gaps for Geminga, PSR B0656+14, and PSR B1055-52 are about $70 \%$ of the outer magnetospheres. This means that the outer gaps of these pulsars are thick. However, the observed spectrum for PSR B0656+14 is not available yet (Ramanamurthy et al. 1996), so we apply our model results only to Geminga and to PSR B1055-52. Figures 6 and 7 compare the model results with the observed data for these two $\gamma$-ray pulsars, and the deduced parameters are given in Table 2. It can be seen from Table 1 that the mature pulsars with longer ages have thick outer gaps, except PSR $\mathrm{B} 1951+32$.

We also apply our model to the mature pulsars with medium outer gaps (Vela, PSR B1706-44, and PSR $\mathrm{B} 1951+32)$. The model results and deduced parameters are

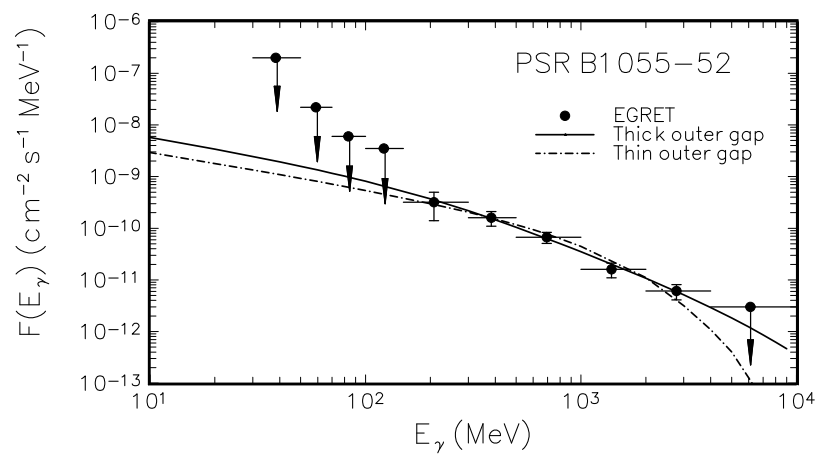

FIG. 7.-Comparison of model results of PSR B1055-52 with observed data by EGRET (circles) (Fierro 1995). The solid curve represents the model result of the thick outer gap, and the dash-dotted curve represents the model result of the thin outer gap, which is taken from Cheng \& Ding (1994).

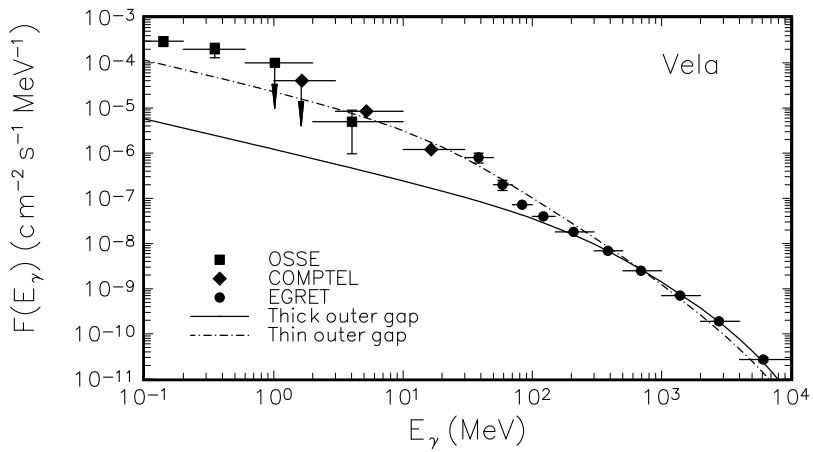

Fig. 8.-Comparison of model results of Vela pulsar with observed data by OSSE (squares) (Strickman et al. 1996), COMPTEL (diamonds) (Bennett et al. 1994), and EGRET (circles) (Kanbach et al. 1994). The solid curve represents the model result of the thick outer gap, and the dashdotted curve represents the model of the thin outer gap, which is taken from Cheng \& Ding (1994).

shown in Figures 8, 9, and 10 and Table 2. From Table 2, it seems that the solid angles of the $\gamma$-ray beaming for these pulsars are relatively smaller. Our model results can fit the observed data, except the Vela pulsar. For the Vela pulsar, our model result does not fit the low-energy part of the spectrum very well because the low-energy break of the spectrum for the Vela pulsar in our model is $\sim 100 \mathrm{MeV}$. In order to fit the observed data of the Vela pulsar, there are two possibilities. One is that $x_{\max }$ is larger, which means that the curvature radius of the top field line in the outer gap becomes larger for some reason. For example, if the particle energy density is comparable to the local magnetic energy density, the field lines will straighten out and $s$ will become larger. We find that the Vela spectrum can be fit by our model if $x_{\max }=4.0$. Another possibility is that the

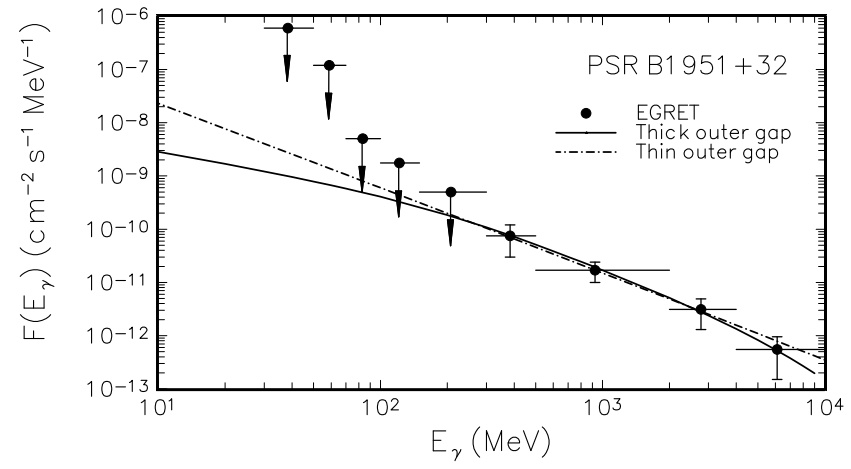

FIG. 9.-Comparison of model results of PSR B1951+32 with observed data by EGRET (circles) (Ramanamurthy et al. 1995). The solid curve represents the model result of the thick outer gap, and the dashdotted curve represents the model of the thin outer gap, which is taken from Cheng \& Ding (1994).

TABLE 2

Deduced Parameters of Five $\gamma$-Ray Pulsars

\begin{tabular}{|c|c|c|c|c|c|}
\hline Pulsar & $\begin{array}{l}\text { Distance } \\
\quad(\mathrm{kpc})\end{array}$ & $N_{0}\left(\mathrm{~cm}^{-3}\right)$ & $\begin{array}{l}\Delta \Omega \\
(\mathrm{sr})\end{array}$ & $X_{\min }$ & $X_{\max }$ \\
\hline PSR B1951 + $32 \ldots \ldots$ & $2.5_{-1.0}^{+0.6}$ & $5.3 \times 10^{29}$ & 2.4 & 0.55 & 2.0 \\
\hline PSR B1706-44...... & $1.8_{-0.5}^{+0.7}$ & $1.4 \times 10^{30}$ & 0.54 & 0.62 & 2.0 \\
\hline PSR B0833-45 ...... & $0.5 \pm 0.1$ & $1.5 \times 10^{30}$ & 1.0 & 0.70 & 2.0 \\
\hline Geminga ............. & $0.16_{-0.03}^{+0.06}$ & $1.2 \times 10^{30}$ & 3.9 & 0.70 & 2.1 \\
\hline PSR B1055-52 ..... & $1.5 \pm 0.4$ & $9.3 \times 10^{30}$ & 1.3 & 0.55 & 2.1 \\
\hline
\end{tabular}




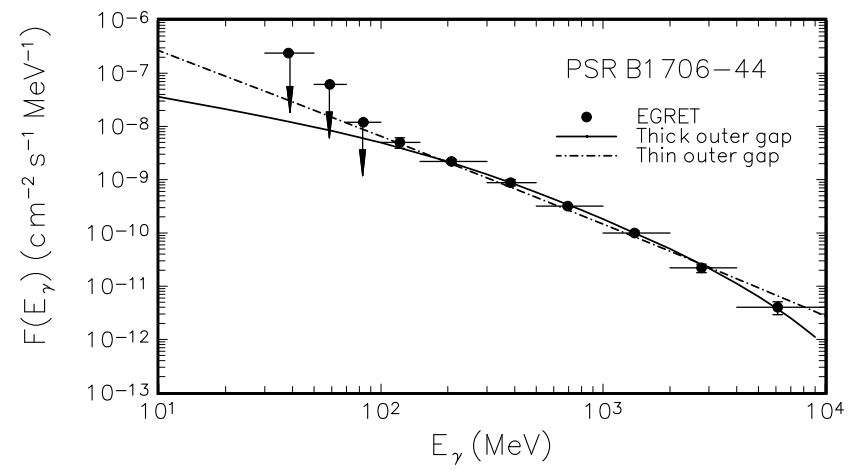

FIG. 10.-Comparison of model results of PSR B1706-44 with observed data by EGRET (circles) (Fierro 1995). The solid curve represents the model result of the thick outer gap, and the dash-dotted curve represents the model of the thin outer gap that is taken from Cheng \& Ding (1994).

observed radiation from the Vela pulsar comes from the radiation of the secondary $e^{ \pm}$pairs; in other words, it should be explained in terms of the thin outer gap model (CHR II).

\section{CONCLUSIONS AND DISCUSSION}

We have proposed the thick outer gap model to explain the observed spectra from $\gamma$-ray pulsars. The main difference between the thick outer gap model proposed here and the thin outer gap model (CHR I and CHR II) is that the observed radiation is produced by different $e^{ \pm}$pairs. For the former, observed radiation is produced by the synchrocurvature radiation of the primary $e^{ \pm}$pairs within the outer gap. For the latter, the observed radiation comes from the secondary $e^{ \pm}$pairs. In order to compare these two models, we have also shown the expected results of the thin outer gap model (Cheng \& Ding 1994) for the five $\gamma$-ray pulsars in Figures 6-10. It is clear that these two models can fit the observed data equally well, except the Vela pulsar. For the Vela pulsar, it can be fitted only by the thin outer gap model, unless the radius of the curvature is larger than the dipolar form near the light cylinder. One of the simplest criteria to differentiate these two models is the luminosity of the low-energy photons. In order to fit the observed spectrum by the thin outer gap model (Cheng \& Ding 1994), the pitch angle $\alpha_{s}$ of the secondary pairs must be sufficiently small to ensure that the low-energy spectral break of the $\gamma$-ray spectrum is greater than $1 \mathrm{MeV}$. Since $\sin \alpha_{s} \sim \lambda / s \sim 10^{-3}$ by fitting the observed data, where $\lambda$ is the mean free path and $\lambda \sim\left(n_{\mathrm{IR}} \sigma_{\gamma \gamma \rightarrow e^{ \pm}}\right)^{-1}$, the implied IR luminosity is $L_{\mathrm{IR}} \sim n_{\mathrm{IR}} \Delta \Omega R_{L}^{2} c E_{\mathrm{IR}}^{ \pm} \sim 3$ $\times 10^{34}\left(P^{-1}\right)(\Delta \Omega / 1 \mathrm{sr})\left(E_{\mathrm{IR}} / 0.01 \mathrm{eV}\right)$ ergs $\mathrm{s}^{-1}$. Such a high intensity of IR photons should be detectable, at least, for the Geminga pulsar, which is located only 160 pc away.

As mentioned above, the parameters $x_{\min }$ and $x_{\max }$ are important in the thick outer gap, especially $x_{\max }$. Generally, $x_{\max }$ should be larger for a mature pulsar with a thick outer gap. Furthermore, the choice of $x_{\min }$ and $x_{\max }$ should be correlated to the phase separation and pulse shape. In fact, from the observations, the pulsars with a thick outer gap (Geminga, PSR B1055-52) have larger phase separations than the Vela pulsar. Based on this argument, our choice for mature pulsars (see Table 2) seems reasonable.

This work is partially supported by a RGC grant from the government of Hong Kong.
Arons, J., \& Scharlemann, E. T. 1979, ApJ, 231, 854

Baym, G., \& Pines, D. 1971, Ann. Phys., 66, 816

Becker, W. 1995, in Ann. NY Acad. Sci., 759, 17th Texas Symposium on

Relativistic Astrophysics and Cosmology, ed. H. Böhringer, G. Morfil, \& J. Trümper, 250

Becker, W., \& Trümper, J. 1993, Nature, 365, 528

.1997, A\& A, in press

Bennett, K., et al. 1993, in Proc. 23d Int. Cosmic Ray Conf. (Calgary), 1, 172

. 1994, ApJS, 90, 823

Caraveo, P. A., Bignami, G. F., Mignani, R., \& Taff, L. G. 1996, ApJ, 461, L91

Chen, K., \& Ruderman, M. 1993, ApJ, 402, 264

Cheng, K. S. 1994, in Proc. Towards a Major Atmospheric Cherenkov Detector III for TeV Astro/Particle Physics, ed. T. Kifune (Tokyo: Universal Academy), 25

Cheng, K. S., Chau, W. Y., Zhang, J. L., \& Chau, H. F. 1992, ApJ, 396, 135

Cheng, K. S., \& Ding, W. K. Y. 1994, ApJ, 431, 724

Cheng, K. S., Ho, C., \& Ruderman, M. A. 1986a, ApJ, 300, 500 (CHR I) .1986b, ApJ, 300, 522 (CHR II)

Cheng, K. S., \& Wei, D. M. 1995, ApJ, 448, 281

Cheng, K. S., \& Zhang, J. L. 1996, ApJ, 463, 271 (Paper I)

Cheng, K. S., Zhang, J. L., \& Zhang, L. 1996, in preparation (Paper II)

Chiang, J., \& Romani, R. W. 1992, ApJ, 400, 629

. 1994, ApJ, 436, 754

Chong, N., \& Cheng, K. S. 1993, ApJ, 417, 219

Fierro, J. M. 1995, Ph.D. thesis, Stanford Univ.

Goldreich, P., \& Julian, W. H. 1969, ApJ, 157, 869

Halpern, J. P., \& Ruderman, M. A. 1993, ApJ, 415, 286

\section{REFERENCES}

Halpern, J. P., \& Wang, F. Y.-H. 1997, ApJ, 477, 905

Harding, A. K. 1981, ApJ, 245, 267

Ho, C. 1989 , ApJ, 342, 369

Kanbach, G., et al. 1994, A\& A, 289, 855

Krausse-Polstorff, J., \& Michel, C. F. 1985, MNRAS, 213, 43P

Mayer-Hasselwander, H. A., et al. 1994, ApJ, 421, 276

Morrison, R., \& McCammon, D. 1983, ApJ, 270, 119

Nel, H. I., et al. 1996, ApJ, 465, 898

Ögelman, H. 1993, in Lives of Neutron Stars, ed. M. A. Alpar, Ü. Kiziloglu, \& J. van Paradijs (Dordrecht: Kluwer), 101

Page, D. 1994, ApJ, 428, 250
.1995, ApJ, 442, 273

Pines, D., \& Alpar, M. A. 1992, in The Structure and Evolution of Neutron Stars, ed. D. Pines, R. Tamagaki, \& S. Tsuruta (Redwood City: AddisonWesley), 7

Ramanamurthy, P. V., et al. 1995, ApJ, 447, L109

. 1996, ApJ, 458, 755

Ruderman, M., \& Cheng, K. S. 1988, ApJ, 335, 306

Ruderman, M. A., \& Sutherland, P. 1975, ApJ, 196, 57

Sauls, J. A. 1989, in Timing Neutron Stars, ed. H. Ögelman \& E. P. J. van den Heuvel (Dordrecht: Kluwer), 457

Shibazaki, N., \& Lamb, F. K. 1989, ApJ, 346, 808

Sturrock, P. 1971, ApJ, 164, 529

Strickman, M. S., et al. 1996, ApJ, 460, 735

Tsuruta, S. 1992, in The Structure and Evolution of Neutron Stars, ed. D. Pines, R. Tamagaki, \& S. Tsuruta (Redwood City: Addison-Wesley), 371

Wambach, J., Ainsworth, T. L., \& Pines, D. 1991, in Neutron Stars: Theory and Observation, ed. D. Pines \& J. Ventura (Dordrecht: Kluwer), 37 